\title{
Multiple Visual Feature Identification and Integration for 3D Recovery of Rotating Objects
}

\author{
Tetsuo Ohtsuka, Jiang Yu Zheng, Kazuaki Tanaka, and Norihiro Abe \\ Faculty of Computer Science and System Engineering, Kyushu Institute of Technology, Iizuk a, Japan 820
}

\section{SUMMARY}

The purpose of this study was to investigate ways to separate and extract various visual information from the continuous images of a rotating object and to recover 3D shapes by integrating the results. In this study, the pattern, corners, (contours) and highlights in an image are separated and extracted through clear qualitative analysis of the visual features. The extracted visual features are successively applied to the "shape from $X$," and the results obtained are integrated. Initially, the features that can be acquired with stability are applied to the shape from $X$, and the shape is recovered. Then, based on the information obtained, ambiguous visual information is determined. By iterating this procedure, ambiguous information is converted to clear explicit information, making it possible to derive a more exact 3D shape. (C) 1998 Scripta Technica, Syst Comp Jpn, 29(13): 56-65, 1998

Key words: Visual features; shape recovery; highlight; 3D model; shape integration.

\section{Introduction}

With the progress of 3D computer graphics technology and the widespread use of virtual reality and multimedia, it is becoming necessary to devise an exact and simple method for inputting a complex 3D shape. As methods of recovery for the $3 \mathrm{D}$ model, projection methods such as the range finder are already used in practice, however the equipment required is expensive. Thus, the authors propose a method based on images with the advantage that 3D shapes can be acquired with low cost. Another advantage is that the image can be transferred over a network, and it is not necessary to provide a near-by recovery system.

Studies of 3D shape recovery using images include studies of the "shape from Xs" $(X=$ contour $[1,2,4,11]$, motion [6, 12-15], highlight $[5,7,9]$, shading $[8,16] \ldots$ ). All of these, however, use recovery from a single kind of visual information, and it is difficult to acquire the precise shape from an arbitrary object in an arbitrary environment. In contrast, the aim of this study was to recover an almost complete 3D model based on multiple kinds of visual information.

In general, formation of an object image is based on two factors: the physical properties inherent to the object (such as shape and reflectivity) and the imaging environment (such as viewpoint, motion and illumination). Thus the image contains various kinds of visual features (such as density, edges, parallax, and highlights). In previous studies, these physical properties and the imaging environment have been simplified and a single kind of shape recovery procedure applied. These approaches, however, lack generality, and cannot fully utilize image data.

Our method uses continuous images input from a single camera, and achieves simple and stable shape recovery of the 3D model under ordinary illumination conditions. The visual features contained in the image possess different unique properties; they must be separated qualitatively, and the shape must be recovered by a corresponding method. Our technique applies the shape from $\mathrm{X}$ to the features that can be acquired with stability according to the physical

CCC0882-1666/98/130056-10

(C) 1998 Scripta Technica 
conditions, and then recovers the shape. By using the features thus obtained, ambiguous features can be determined [3]. By iterating the above procedure, the ambiguous features are converted to explicit features, which helps to acquire a more exact shape. In this paper, various 3D recovery methods for visual information are described and these methods are compared. Then, the integration of shape information is considered based on the characteristics of these methods.

\section{3D Shape Recovery for a Rotating Object}

\subsection{Observation conditions}

Figure 1 shows a system that acquires continuous images from a single camera. In order to recover an object in this system, the following imaging environment is needed.

1. The amount of rotation is known or can be estimated from the images.

2. The axis of the camera is parallel to the rotation plane.

3. The object and the background can be easily separated.

4. The direction of illumination is aligned to the camera direction. In other words, there is no shade on the object surface.

5. The camera is modeled as a normal projection.

The following physical conditions are assumed.

1. There is no constraint on the shape or surface pattern.

2. There can be both specular and diffuse reflection.
3. The object is opaque.

The object is placed on a rotating table. Epipolar plane images from pixel lines at different heights, for which the cross-sections of the object are to be acquired, are obtained during rotation. These continuous images are called epipolar plane images (EPI) [10]. In this study, EPI constructed under the above conditions are considered, and the contours, the pattern on the object surface, the locus of the corners, and highlights are considered.

The viewed pattern on the object surface as well as corners on the object surface are kept invariant to their surface positions, independently of the rotation angle or the view line. Consequently, they are called fixed points. By noting the behavior of a single point, the shape from motion approach $[6,10]$ can be applied. The silhouette of the object against the background, as well as the position of the highlights, change with the rotation angle on the object surface. Consequently, they are called moving points. The shape from contour approach $[1,2,4,11]$ can be applied to the recovery from the contour, and the shape from highlight approach $[5,7]$ can be applied to recovery from highlights.

\subsection{Theory of recovery from contour}

Figure 2 is an overview of Fig. 1. Point $\mathrm{O}$ is the axis of rotation. An orthogonal coordinate system $\mathrm{X}-\mathrm{Z}$ is defined with the point $\mathrm{O}$ as its center. Point $\mathrm{P}$ is a point on the object surface. The point obtained by rotating the point $\mathrm{P}\left(P_{x}, P_{y}\right)$ by an angle $\theta$ is written $\left(P_{x}(\theta), P_{y}(\theta)\right)$. In order to simplify the formulation of the recovery procedure, an equivalent coordinate system is considered, in which the camera rotates around the object.

Point $\mathrm{O}^{\prime}$ is the mapping of point $\mathrm{O}$ to the image plane. $x(\theta)$ is the distance from point $\mathrm{O}^{\prime}$ to the map $\mathrm{p}$ of point $\mathrm{P}$. The unit vector $\vec{x}$ of vector $\mathrm{O}^{\prime} \mathrm{p}$ is given by $(\cos \theta, \sin \theta)$, and the unit vector $\vec{v}$ of the line of sight is given by

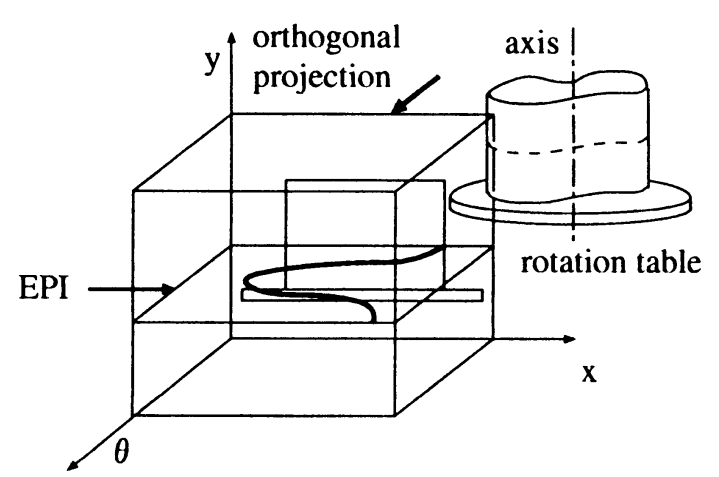

Fig. 1. Image formation geometry of EPI.

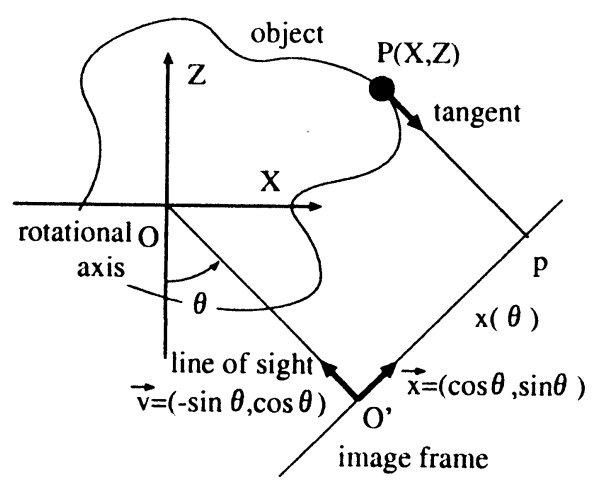

Fig. 2. Shape recovery from contour. 
$(-\sin \theta, \cos \theta)$. Consequently, the distance $x(\theta)$ between points $\mathrm{O}^{\prime}$ and $\mathrm{p}$ is

$$
x(\theta)=\vec{x} \cdot \vec{P}=P_{x}(\theta) \cos \theta+P_{z}(\theta) \sin \theta
$$

Differentiating Eq. (1), the following relation is obtained:

$$
\begin{aligned}
\frac{\partial x(\theta)}{\partial \theta}= & \frac{\partial P_{x}}{\partial \theta} \cos \theta+\frac{\partial P_{z}}{\partial \theta} \sin \theta \\
& -P_{x} \sin \theta+P_{z} \cos \theta
\end{aligned}
$$

The line of sight passing through the contour is a tangent line to the object surface. Consequently, the scalar product between $x$ and the tangent vector is 0 :

$$
\left(\frac{\partial P_{x}}{\partial \theta}, \frac{\partial P_{z}}{\partial \theta}\right) \cdot(\cos \theta, \sin \theta)=0
$$

The following expression for contour recovery is obtained from Eqs. (1) to (3):

$$
\begin{aligned}
& P_{x}=x \cos \theta-\frac{\partial x}{\partial \theta} \sin \theta \\
& P_{z}=x \sin \theta+\frac{\partial x}{\partial \theta} \cos \theta
\end{aligned}
$$

The result of the recovery from the contour is an envelope of lines of sight from all possible angles. Other parts (unknown regions) are not recovered. The unknown regions are recovered by using the property that the locus of the contour is not smooth on EPI $[1,2]$.

\subsection{Theory of recovery from fixed point}

When the point $\mathrm{P}$ is a fixed point, $P_{x}(\theta)$ and $P_{z}(\theta)$ in Eq. (1) are constant. Consequently, the constants $P_{x}$ and $P_{z}$ are determined so that the square sum of the errors $\Sigma\left(P_{x} \cos \theta+P_{z} \sin \theta-x(\theta)\right)^{2}$ is minimized. Then, the following recovery formula for the fixed point is obtained:

$$
\begin{gathered}
\left(\begin{array}{c}
P_{x} \\
P_{z}
\end{array}\right)=\frac{1}{\sum \cos ^{2} \theta \sum \sin ^{2} \theta-\left(\sum \sin \theta \cos \theta\right)^{2}} \\
\times\left(\begin{array}{c}
\Sigma \sin ^{2} \theta \Sigma x \cos \theta-\Sigma \sin \theta \cos \theta \Sigma x \sin \theta \\
-\Sigma \sin \theta \cos \theta \Sigma x \cos \theta+\Sigma \cos ^{2} \theta \Sigma x \sin \theta
\end{array}\right)
\end{gathered}
$$

where $\Sigma$ is $\Sigma_{\theta}$, and $x$ is $x(\theta)$.

\subsection{Theory of recovery from highlights}

By the law of reflection, when the angle of incidence is 0 , highlights are observed at points on the object surface where the normal is in the opposite direction to the illumination. It is not always true that a highlight appears as a continuous locus. Since the scalar product of the illumination direction and the tangent to the point of a highlight is 0 , the following condition is obtained (Fig. 3):

$$
\left(\frac{\partial P_{x}}{\partial \theta}, \frac{\partial P_{z}}{\partial \theta}\right) \cdot(-\sin \theta, \cos \theta)=0
$$

The following differential equations are obtained from Eqs. (1), (2), and (6).

$$
\begin{aligned}
& \frac{\partial P_{x}}{\partial \theta}-\frac{\cos \theta}{\sin \theta} P_{x}=\cos \theta \frac{\partial x}{\partial \theta}-\frac{\cos ^{2} \theta}{\sin \theta} x \\
& \frac{\partial P_{z}}{\partial \theta}+\frac{\sin \theta}{\cos \theta} P_{z}=\sin \theta \frac{\partial x}{\partial \theta}+\frac{\sin ^{2} \theta}{\cos \theta} x
\end{aligned}
$$

Solving Eqs. (7) and (8) for $P_{x}$ and $P_{z}$, the following formulas for highlight recovery are obtained:

$$
\begin{aligned}
P_{x}(\theta)= & \left(\int_{\theta_{i}}^{\theta} Q(\theta) e^{\int_{\theta_{i}}^{\theta} P(\theta) d \theta} d \theta+P_{x \theta_{i}}\right) \\
& \times e^{-\int_{\theta_{i}}^{\theta} P(\theta) d \theta}
\end{aligned}
$$

where, $P(\theta)=-\frac{\cos \theta}{\sin \theta}, Q(\theta)=\cos \theta \frac{\partial x}{\partial \theta}-\frac{\cos ^{2} \theta}{\sin \theta} x$

$$
\begin{aligned}
P_{z}(\theta)= & \left(\int_{\theta_{i}}^{\theta} Q(\theta) e^{\int_{\theta_{i}}^{\theta} P(\theta) d \theta} d \theta+P_{z \theta_{i}}\right) \\
& \times e^{-\int_{\theta_{i}}^{\theta} P(\theta) d \theta}
\end{aligned}
$$

where, $P(\theta)=\frac{\sin \theta}{\cos \theta}, Q(\theta)=\sin \theta \frac{\partial x}{\partial \theta}+\frac{\sin ^{2} \theta}{\cos \theta} x$

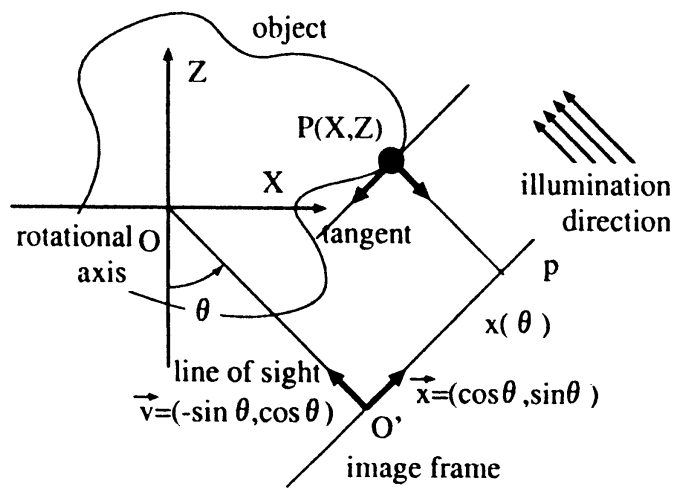

Fig. 3. Shape recovery from highlight. 
$P_{x \theta_{i}}$ and $P_{z \theta_{i}}$ are the initial conditions. An arbitrary known 3D position on the highlight locus gives the initial condition. Usually, the intersection of the highlight locus and the fixed point locus is determined by fixed point recovery, and is used as the initial condition.

\subsection{Comparison of methods}

The recovery methods are summarized in Table 1 . The recovery by the fixed point is calculated by the leastsquares method. Consequently, the accuracy of the recovery is high when the locus is long. The result of recovery, however, is a point. The result of recovery by the contour contains errors that are larger than in recovery by fixed point. The shape is recovered as the envelope of the lines of sight from all possible angles. Consequently, the result of the recovery is a continuous curve.

The recovery by highlights includes an integration. Thus errors accumulate when the locus is long. The accuracy is lower than in recovery by a fixed point or by the contour. On the other hand, it may be the case that a part of the object which cannot be recovered by the contour can be recovered.

\section{Separation of Visual Features}

\subsection{Intensity characteristics of visual features}

The intensity on EPI is proportional to the light reflected from the object. The reflected light contains the diffused reflection component and the specular reflection component and is their sum. The intensity distribution due to reflection at the contour of the pattern is as shown in the upper part of Fig. 4. In the intensity distribution due to highlights, a sharp peak is observed, as in the middle left of Fig. 4, when specular reflection dominates. The peak is flat, as in the middle right of Fig. 4, when specular reflection is weak. The intensity is the sum of these two components, and exhibits the distribution on EPI as shown in the lower part of Fig. 4. diffused reflectance (near texture)

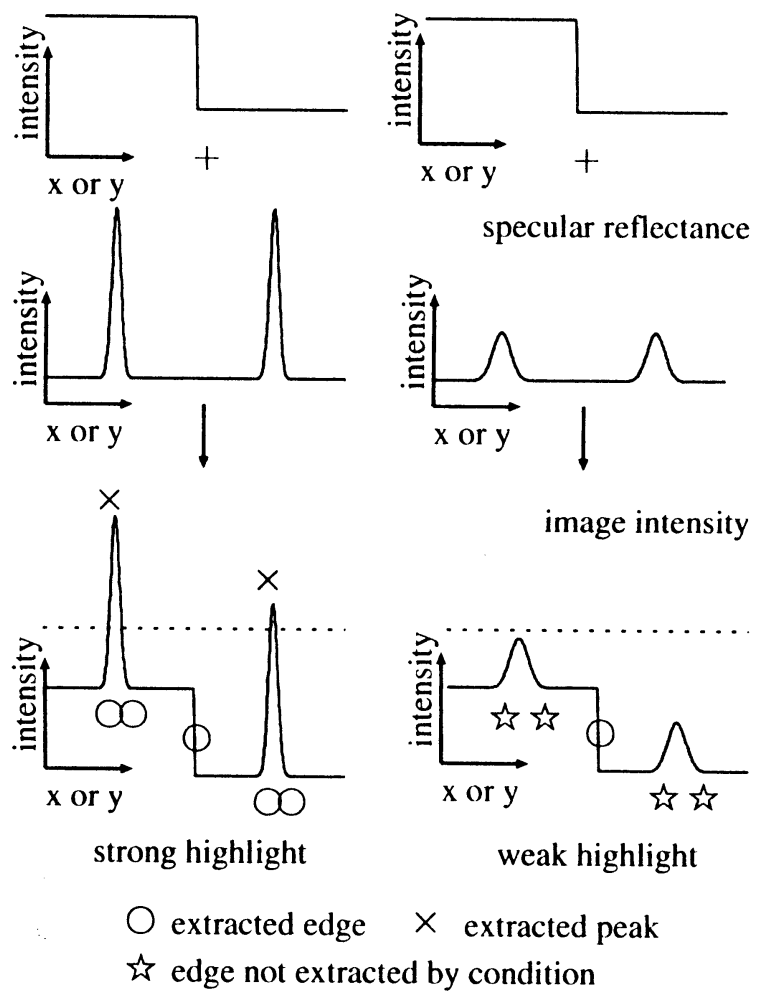

Fig. 4. Image intensity distribution on EPI.

In the case of a strong highlight, it suffices to extract the peak using a threshold. By differentiating the result, edges are detected at both sides of the peak. In the case of a weak highlight, the peak is not detected if it is lower than the threshold. In such cases, the edge may or may not be detected by differentiation. Separation of the visual features based on intensity is incomplete. Consequently, highlights are extracted in this study by considering the tracked and recovered 3D shape.

Table 1. Comparison of recovery methods

\begin{tabular}{|c|c|c|c|c|}
\hline Feature & Condition & Result of recovery & Formula & Error \\
\hline fixed point & $\begin{array}{l}\text { pattern or corner on object } \\
\text { surface }\end{array}$ & point & first-order derivative & small \\
\hline contour & background can be extracted & envelope & least-square method & medium \\
\hline highlight & $\begin{array}{l}\text { parallel ray with known } \\
\text { direction of illumination }\end{array}$ & convex/concave curve & $\begin{array}{l}\text { first-order differential } \\
\text { equation }\end{array}$ & large \\
\hline
\end{tabular}




\subsection{Loci of visual features on EPI}

The locus of the fixed point forms a sinusoidal function with respect to the angle of rotation. It can be detected from varying angles. Figure 5 is the result of simulation of the EPI locus for shapes with various curvatures. The thin line is (b) is the locus of the point marked $\mathrm{X}$ in (a). The thick line is the locus of the highlight observed in that case. As is seen from Fig. 5, the fixed point loci do not cross on the EPI.

The highlight locus changes as follows according to the curvature of the object surface, as shown in Fig. 6 [5, 9].

- The locus is not observed in the region with infinite positive curvature.

- For a region with positive curvature, the velocity on the image is lower than that of the fixed point locus.

- The locus follows a horizontal line in the region with zero curvature. In the region with negative curvature, the velocity on the image is greater than that of the fixed point locus.

On the EPI, the highlight loci never cross, but there may be an intersection between the locus of the pattern on the object surface, a kind of fixed point locus, and the highlight locus. This implies that the highlights and the pattern on the object surface can be observed at the same

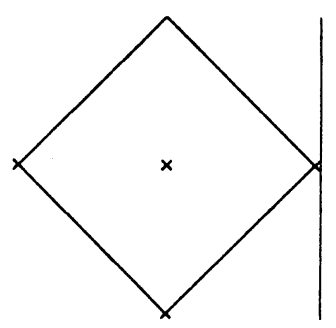

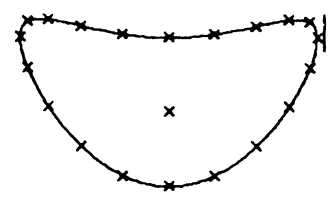

(a)

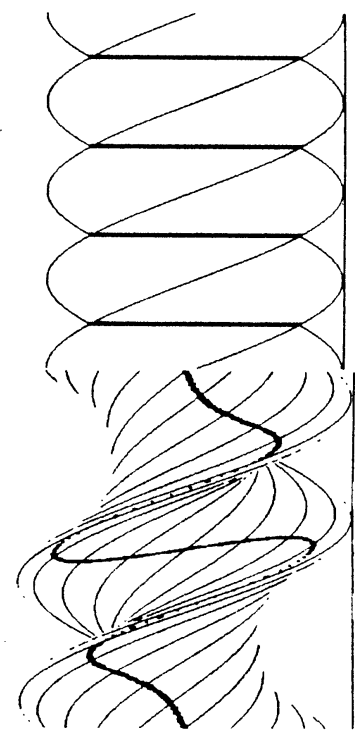

(b)
Fig. 5. Simulated EPI (a) shape, (b) traces of fixed points and highlights.

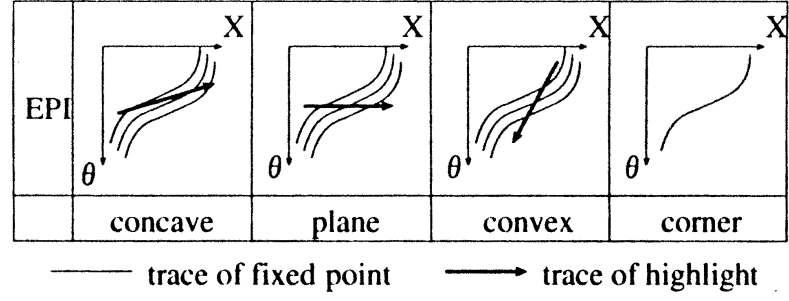

Fig. 6. Trajectories of highlight and fixed points of different shapes.

time from a certain line of sight. In this study, such differences among the motions of the features are recognized and the features observed on the EPI are separated.

\subsection{Method for separation of image features}

There are various mixed features on the EPI. If the extracted feature is incorrectly applied to the recovery formula, a false result is obtained. Consequently, in this study, the features are separated by the following qualitative method based on the intensity, the locus, and the behavior in 3D space of the features.

The locus of the contour can be detected with stability by scanning the background. At a point with a highlight on the EPI, the intensity is much higher than that of a point without a highlight. Consequently, the highlight is detected as a point that has an intensity greater than that of the surroundings.

When the first-order derivative of the EPI with respect to the angle of rotation $\theta$ is formed, we obtain two mixed kinds of parallel lines on either sides of the fixed point locus and the highlight locus. By deleting the previously extracted contour locus and highlight locus from the above image, the fixed point locus is extracted. In our experiments, the difference of intensities between the locus images is calculated, in order to eliminate the locus.

In order to examine whether or not the extracted result is correct, we determine whether or not the properties described in section 3.2 are satisfied. We also examine whether or not the locus forms a sinusoidal function. The procedures are summarized in Fig. 7.

\section{Integration of Results}

The results are integrated by considering the properties of the recovery methods from various features, so that the shape can be recovered with the highest accuracy. As discussed in section 2.5 , the error of the recovery decreases in the order of: recovery from a fixed point, recovery from 


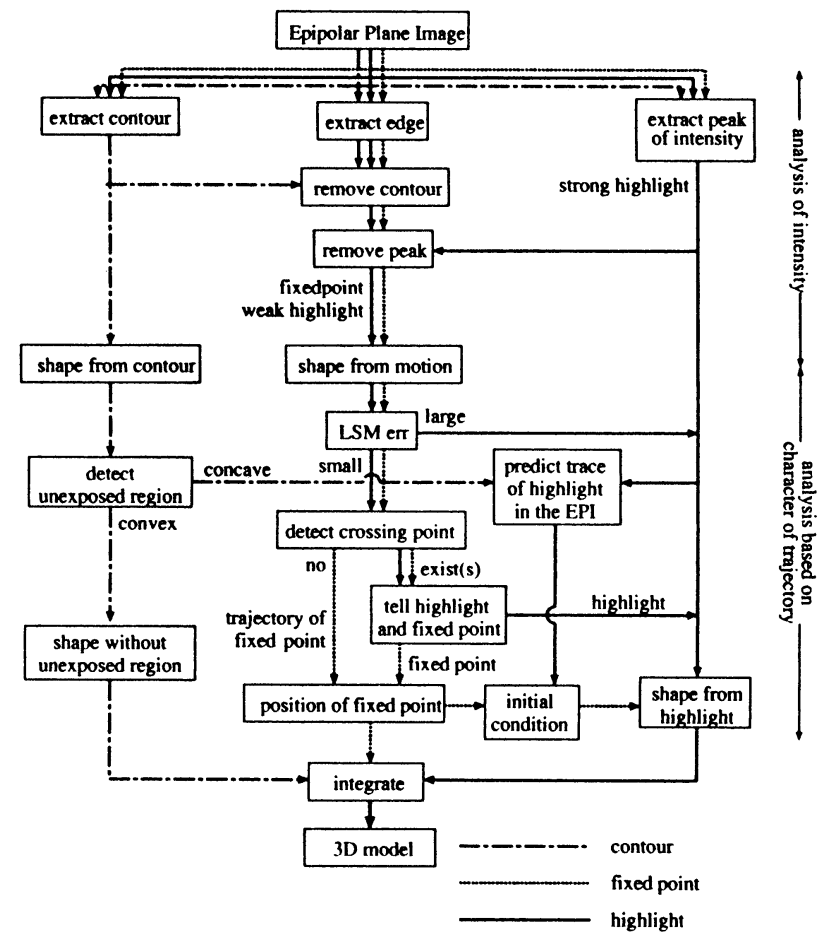

Fig. 7. Process of 3D recovery.

the contour, and recovery from highlights, but the constraints on the shape that can be recovered increase in this order. In the case of highlight recovery, the accuracy is high when the locus is short. Consequently, the highlight locus is separated at the intersection with the fixed point locus. Using the 3D position of the fixed point at the intersection as the initial value, highlight recovery is applied to the segment. This improves the accuracy of the recovery.

Based on the above, the result of recovery from the contour is integrated with recovery from a fixed point. The unknown region is interpolated based on the results of highlight recovery or by straight-line interpolation. When the result shown in Fig. 8 is obtained, in which there are results from the fixed point method and the contour method, such as points $\mathrm{A}, \mathrm{F}$, and $\mathrm{G}$, the result by the contour method is modified so that the results by the contour method and

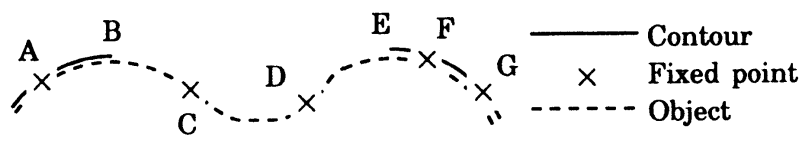

Fig. 8. Integration of contour and fixed points. the fixed point method agree. In other words, the results are integrated. The result for the same point may not agree due to recovery error. Consequently, the points with error (below a certain threshold) are regarded as a single point. In regions such as B-C-D-E, where there are no results from the contour, the region is interpolated based on the results from highlights. In other words, not all of the results by highlights are used. If a highlight locus is not observed, straight-line interpolation is applied.

\section{Experimental Results}

\subsection{System configuration}

An experiment was performed using the system shown in Fig. 9. The distance between the object and the camera was 3 to $4 \mathrm{~m}$. An object more than $10 \mathrm{~cm}$ high was recovered. A lens with long focal length was used with the camera, and the system can be considered to provide normal projection.

\subsection{Recovery example (1)}

Consider Fig. 10(a). Figure 10(b) shows an EPI. Figure 10(c) shows the result of contour extraction from Fig. 10(b). Figure 10(c) shows the result of peak extraction from Fig. 10(b), where the highlight is extracted. Figure 10(e) shows the result of differentiation and peak extraction of Fig. 10(b). Included are two mixed parallel loci for the fixed point locus, the contour locus and the highlight locus. Eliminating the previously extracted highlight and contour loci, the result shown in Fig. 10(f) is obtained. This contains only the fixed point.

After thus separating the loci, we determine whether or not the loci are correctly separated. Then, the respective recovery methods are applied. Figure 10(g) shows the result obtained by contour recovery. Figure 10(h) shows the result of fixed point recovery. Figure 10(i) shows the result when the unknown region of Fig. 10 (g) is recovered by highlight recovery. Figure 10(j) shows the result of integrating Figs. $10(\mathrm{~g})$ to $10(\mathrm{i})$. Figures $10(\mathrm{k})$ and $10(\mathrm{l})$ shows the results of

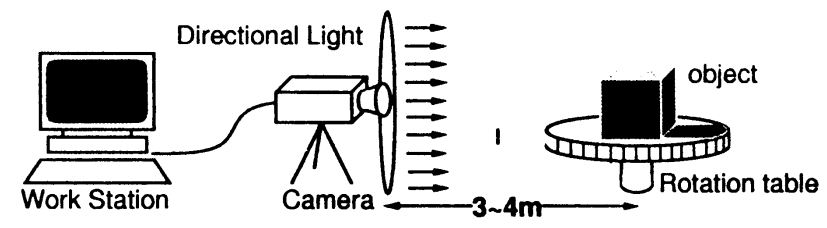

Fig. 9. System environment. 


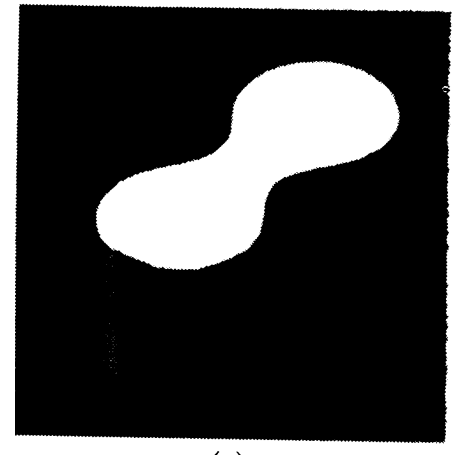

(a)

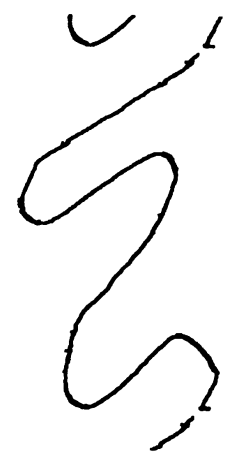

(d)

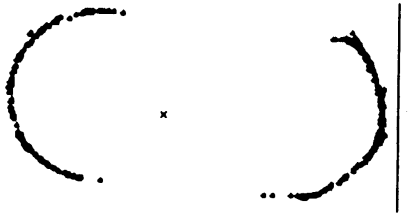

(g)

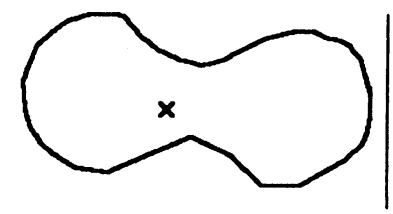

(j)

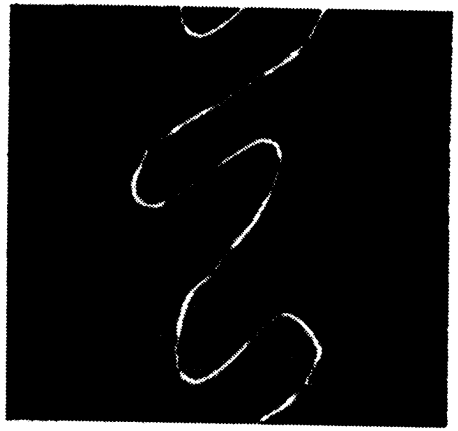

(b)

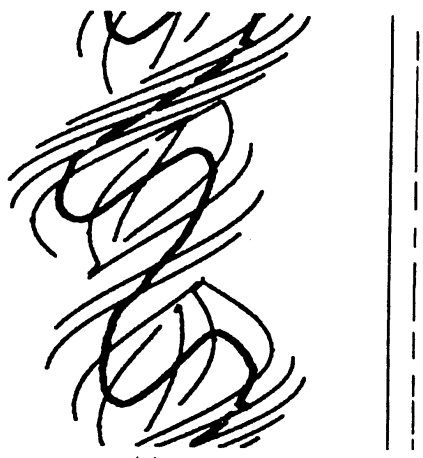

(e)

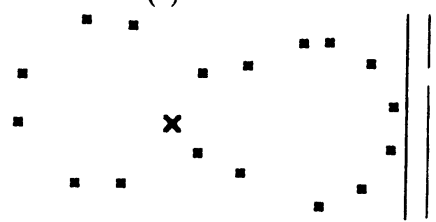

(h)

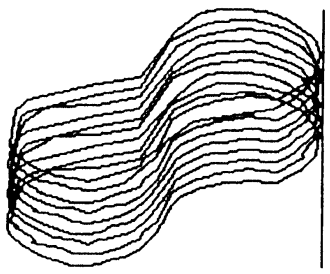

(k)
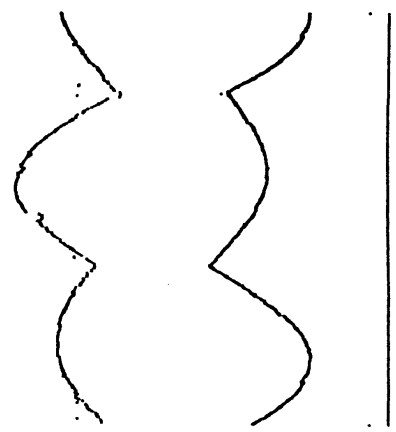

(c)

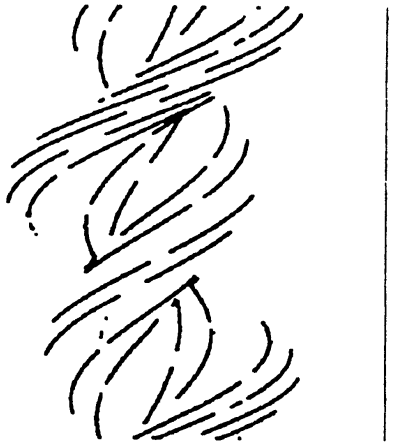

(f)

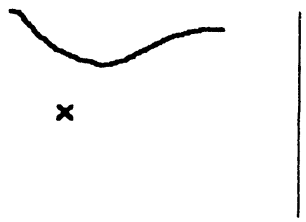

(i)

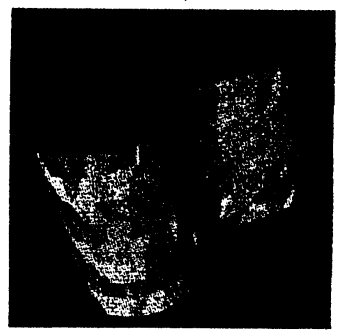

(1)

Fig. 10. Experiment 1 (a) Object, (b) EPI, (c) contour, (d) extracted highlight, (e) edge image, (f) remove contour and highlight from (e), (g) estimated shape from contour, (h) estimated shape from fixed point, (i) estimated shape from highlight, (j) integrated shape, (k) 3D model constructed by connecting shapes at all rotation planes, (l) estimated 3D model.

integrating the cross-sections at various heights; they are shown superposed.

\subsection{Recovery example (2)}

Recovery processing was applied to the object shown in Fig. 11(a). Figure 11(b) shows the EPI for a certain height. Figures 11(d) and 11(e) show the results of extraction and recovery of the contour and the fixed point, respectively. Figure 11(f) shows the result of integration. The highlight appears only for a short period, resulting in a short locus in EPI. Consequently, less of the shape is recovered from the highlight and used in the integration. Figure 11(c) shows the shape obtained. 


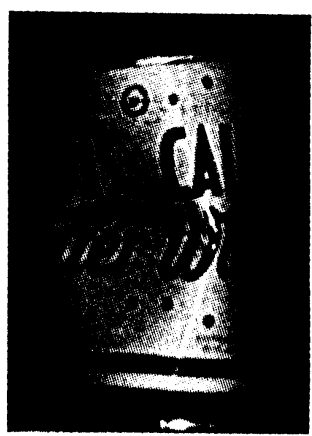

(a)

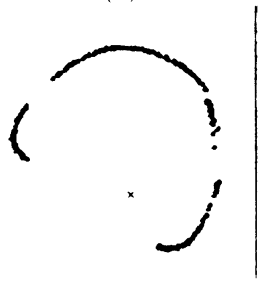

(d)

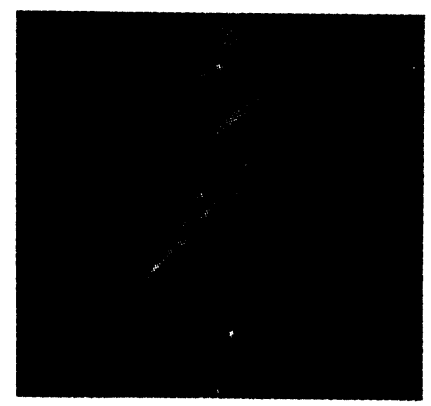

(b)

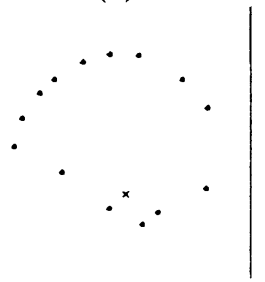

(e)

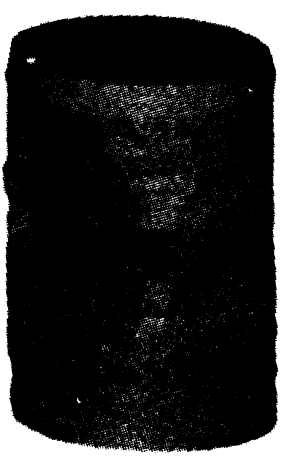

(c)

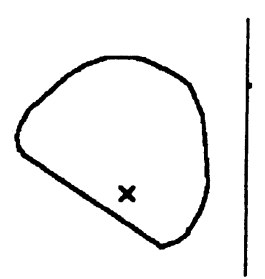

(f)

Fig. 11. Experiment 2 (a) Object, (b) EPI, (c) estimated 3D model, (d) estimated shape from contour, (e) estimated shape from fixed point, (f) integrated shape.

\subsection{Recovery example (3)}

Recovery processing was applied to the object shown in Fig. 12(a). Figure 12(b) shows the EPI at a certain height.
Figures 12(d) to 12(f) show the results of extracting the contour, the fixed point and the highlights, respectively. Figure 12(g) shows the result of integration. Figure 12(c) shows shape obtained.

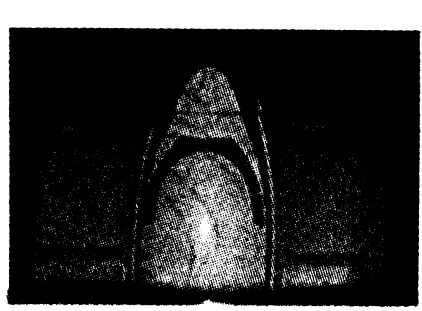

(a)

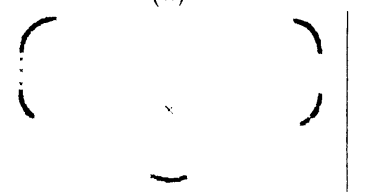

(d)

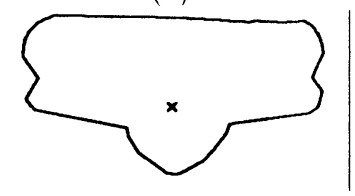

(g)

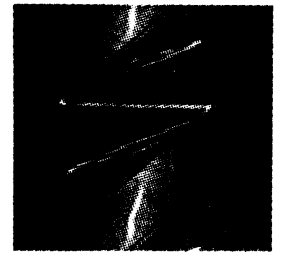

(b)

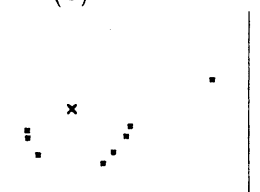

(e)

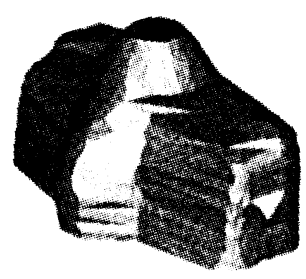

(c)

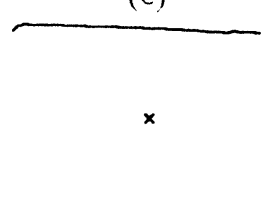

(f)

Fig. 12. Experimental 3 (a) Object, (b) EPI, (c) estimated 3D model, (d) estimated shape from contour, (e) estimated shape from fixed point, (f) estimated shape from highlight, (g) integrated shape. 


\section{Conclusions}

This paper has analyzed the properties of various features that coexist in continuous images of a rotating object. The fixed point, the contour and the highlights are qualitatively separated based on information that can be extracted with stability. The results are integrated to provide a cross-sectional shape for each height. Then, by superposing the cross-sections, the 3D shape is acquired.

Further studies are required of methods that can separate the loci quantitatively by setting the parameters in the image processing, as well as methods that recover the shape using intensity information in the region where straight line interpolation is applied in the integration. The proposed method requires only a simple installation and environment for the measurements. Consequently, it is expected that the method will be widely applied in simple recovery methods for objects, and used for low-cost 3D model recovery in developing multimedia systems.

Acknowledgments. This study was supported in part by a grant from the Okawa Information Communication Foundation as well as a grant-in-aid, of the Ministry of Education.

\section{REFERENCES}

1. J.Y. Zheng. Acquiring 3D models from sequences of contours. IEEE PAMI, 16, No. 2, pp. 163-178 (Feb. 1994).

2. J.Y. Zheng and F. Kishino. Recovery of 3D model by continuous silhouette and detection of unknown region. Trans. I.E.I.C.E. (D-II), J76, No. 6, pp. 11141122 (June 1993).

3. J.Y. Zheng and F. Kishino. Verifying and combining different visual cues into a complete 3D model. CVPR92, pp. 777-780 (1992).

4. J.Y. Zheng and F. Kishino. 3D model from contours: further identification of unexposed areas. 11th ICPR, 1, pp. 349-353 (1992).
5. J.Y. Zheng, Y. Fukagawa, T. Ohtsuka, and N. Abe. Acquiring 3D models from rotation and a highlights. 12th ICPR, pp. 349-353 (1994).

6. J.Y. Zheng, H. Kakinoki, K. Tanaka, and N. Abe. Acquiring 3D models from fixed points during rotation. 3rd ICAR, 1, pp. 459-463 (1994).

7. J.Y. Zheng, Y. Fukagawa, and N. Abe. Shape and model from specular motion. 5th ICCV, pp. 72-79 (1995).

8. J. Lu and F. Little. Reflectance function estimation and shape recovery from image sequence of rotating object. 5th ICCV, pp. 80-86 (1995).

9. Y. Fukagawa, K. Tanaka, J.Y. Zheng, and N. Abe. Acquisition of 3D shape by highlight detection. Trans. I.E.I.C.E. (D-II), J79, No. 5, pp. 719-726 (May 1996).

10. H. Baker and R. Bolles. Generalizing epipolar-plane image analysis on the spatiotemporal surface. CVPR-88, pp. 2-9 (1988).

11. R. Vaillant and O.D. Faugeras. Using extremal boundaries for 3-D object modeling. IEEE Trans. PAMI, 14, No. 2, pp. 157-173 (Feb. 1992).

12. T. Kaneko. 3D shape input by quasi-static rotation of object. Tech. Rep. I.E.I.C.E., PRL85-22 (1985).

13. M. Yamamoto. 3D shape input from continuous stereo images. Trans. I.E.I.C.E. (D), J69, No. 11, pp. 1631-1638 (Nov. 1986).

14. T. Yasuno and S. Susuki. Surface structure recovery by hidden part analysis of spatio-temporal image. Trans. Inf. Proc. Soc., 34, No. 10, pp. 2174-2183 (1993).

15. S. Hiura, K. Sato, and M. Iguchi. Simultaneous measurement of shape and reflectivity by rotating object. Trans. Inf. Proc. Soc., 36, No. 10, pp. 2295-2302 (1995).

16. J.Y. Zheng, H. Kakinoki, K. Tanaka, and N. Abe. Computing 3D models of rotation object from moving shading. 13th ICPR, 1, pp. 800-804 (1996).

17. A. Murata, J.Y. Zheng, Y. Fukagawa, and N. Abe. Acquisition of 3D shape of mirror reflecting object by multiple light sources. Trans. I.E.I.C.E. (D-II), J80, No. 7, pp. 1659-1667 (June 1997). 


\section{AUTHORS (from left to right)}
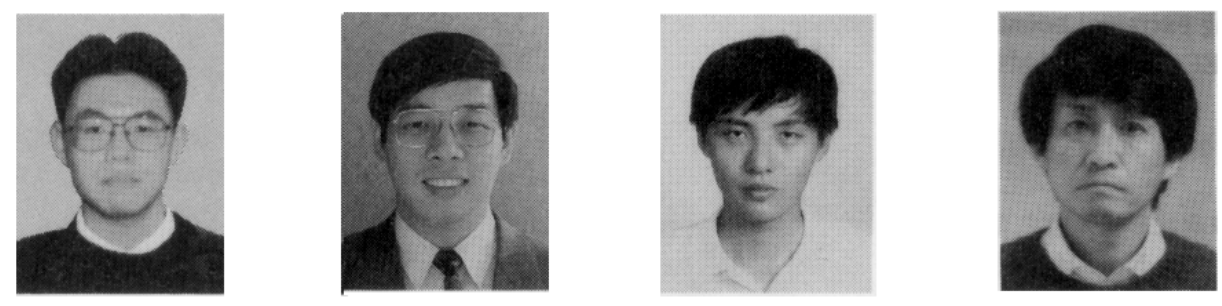

Tetsuo Ohtsuka graduated in 1994 from Kyushu Institute of Technology. Completed first half of doctoral program (Information Systems) in 1996, and joined Mitsubishi Electric Corporation.

Jiang Yu Zheng (member) graduated in 1983 from Fudan University, China. Admitted to graduate school, became visiting student at Osaka University. Completed M.S. program in 1987 and doctoral program in 1990, Osaka University. D.Eng. Researcher, ATR Communication Systems Research Laboratories. Associate professor, 1993, Kyushu Institute of Technology. Research Award, 1991, Information Processing Society. Engaged in research on 3D image processing and robot vision.

Kazuaki Tanaka (member) graduated in 1991 from Kyushu Institute of Technology. Completed M.S. program (Information Science) in 1993, became research associate, Faculty of Information Engineering. Member, Information Processing Society, Japan Robotics Society, Japan Society for Virtual Reality.

Norihiro Abe (member) graduated in 1969 from Osaka Univ. Completed doctoral program in 1974. Associate professor, Osaka University. Since 1991, Professor, Kyushu Institute of Technology. D.Eng. Engaged in research on artificial intelligence, especially applications of AI techniques to mechanical systems. In addition, engaged in research on integration of language and image understanding and on virtual reality. Author/translator of "Introduction to Prolog programming" (Kyoritsu Press), "LISP" (Baifukan Press) and "Prolog and AI" (Kindaikagaku Press). Member, Society for Artificial Intelligence, and AAAI. 